\title{
土工合成材料加筋土挡墙运行期筋材拉力塑性 力学分析方法
}

\author{
刘华北 ${ }^{1 *}$ ，王春海 ${ }^{1}$, 汪䂞 ${ }^{2}$ \\ 1. 华中科技大学土木与水利工程学院, 武汉 430074; \\ 2. 江西理工大学软件工程学院, 南昌 330031 \\ *E-mail: hbliu@hust.edu.cn
}

收稿日期: 2021-07-12; 接受日期: 2021-09-01; 网络版发表日期: 2022-04-08

国家自然科学基金(批准号: 51778259)资助项目

\begin{abstract}
摘要 土工合成材料加筋土挡墙结构具备优越的力学性能, 与此同时, 该类支挡结构可大大降低水泥及钢筋用量, 从而降低碳排放, 对于“碳达峰”“碳中和”具有重要意义. 相较于其他支挡结构, 加筋土挡墙结构历史较短, 现有设 计规程中挡墙的设计以稳定校核为主, 但加筋土挡墙为柔性结构, 其运行期的服役性能非常关键. 本文总结了本 研究团队10多年来在加筋土挡墙筋材拉力分析方面的研究成果，建立了一套正常荷载作用下筋材拉力的塑性力 学分析方法. 该方法以加筋土潜在内部滑移面处的筋土协调变形为基础, 充分考虑填土非线性应力应变关系、填 土塑性变形、筋材长期刚度、主动区填土的力学平衡以及面层的力学平衡, 可比较合理地估算直立及倾斜面板 加筋土挡墙在运行期的筋材拉力. 本文以文献中的加筋土挡墙筋材拉力实测结果和数值模拟分析的结果验证了 筋材拉力塑性力学分析方法的有效性.
\end{abstract}

关键词土工合成材料, 加筋土挡墙, 服役性能, 筋材拉力, 塑性理论

\section{1 引言}

加筋土技术历史悠久, 早在 5000 多年前, 我国的良 渚先民们即应用草裹泥技术筑坝，而我国的长城建设 更是大量采用了植物枝秆加筋技术 ${ }^{[1,2]}$. 不过，现代意 义的土工合成材料加筋土(geosynthetic-reinforced soil, GRS)结构出现于 20 世纪70 80年代，国内外的工程师 们应用高分子聚合物制造土工合成材料(主要包括土 工格栅、土工织物以及土工加筋带)，用于加固土体， 建造了大量的加筋土挡墙和加筋土边坡等土工结构
物. 国内外 40 多年的工程实践表明, 土工合成材料加筋 土结构具备很多优点. 首先, 跟其他相同功用土工结构 相比, 土工合成材料加筋土结构用料省、经济性强. 其 次, 加筋土结构为柔性结构, 适应变形能力强, 特别适 用于软土地区基础设施建设. 其三, 国内外大量的地震 灾后调查表明，土工合成材料加筋土结构具备优越的 抗震性能. 其四, 应用加筋土技术建造土工结构, 可以 大大降低水泥、钢筋等的用量，降低碳排放，对于“碳 达峰”“碳中和”具有重要意义 ${ }^{[3,4]}$. 鉴于上述优点, 土工 合成材料加筋土结构在我国基础设施建设中具有巨大

引用格式: 刘华北, 王春海, 汪否. 土工合成材料加筋土挡墙运行期筋材拉力塑性力学分析方法. 中国科学: 技术科学, 2022, 52: 1083-1095 Liu H B, Wang C H, Wang L. Analyzing operational reinforcement loads of geosynthetic-reinforced soil retaining walls based on plasticity theory (in Chinese). Sci Sin Tech, 2022, 52: 1083-1095, doi: 10.1360/SST-2021-0287 
的应用潜力.

加筋土挡墙是最早，也是最主要的土工合成材料 加筋土结构形式. 加筋土挡墙的基本结构形式如图 1 所示，该类支挡结构的承载能力主要依赖水平铺设的 土工合成材料筋材与填土的相互作用. 加筋土前方的 面层比较薄, 其主要功能是防止填土局部破坏. 面层 的形式多种多样，包括混凝土模块面板、土工合成材 料返包、整体现浇混凝土板、预制钢筋混凝土板、格 宾等. 填土与面层之间需设置排水层. 在国内外绝大多 数设计规程中, 加筋土挡墙的设计主要为强度设计, 包 括内部稳定、外部稳定、地基稳定、整体稳定校核等 内容, 但由于该类支挡结构属于柔性结构, 其服役性能 设计也非常重要，而目前大多数设计规程均未提供合 理的服役性能分析方法 ${ }^{[5 \sim 8]}$.

加筋土挡墙的服役性能可应用数值方法进行分 析. 基于合适的填土本构模型、加筋材料应力-应变时间关系以及筋-土-面板相互作用模型，应用非线性 有限元法或有限差分法可合理地分析加筋土挡墙在正 常工作荷载作用下的筋材拉力及变形, 从而有效地评 估结构的服役性能. 20 世纪 80 年代以来，国内外学者 广泛应用数值模拟方法研究了加筋土挡墙的服役响应 特性 ${ }^{[9 \sim 15]}$, 其中Helwany 和 $\mathrm{Wu}^{[16]}$ 、 Liu等人 ${ }^{[17 \sim 19]}$ 还研究 了筋材及填土蠕变性能对加筋土挡墙服役性能的影 响. 但是, 应用数值方法分析加筋土挡墙服役性能对使 用者的要求比较高, 且模型参数往往比较复杂, 因此, 数值分析方法在加筋土结构设计中的应用并不多.

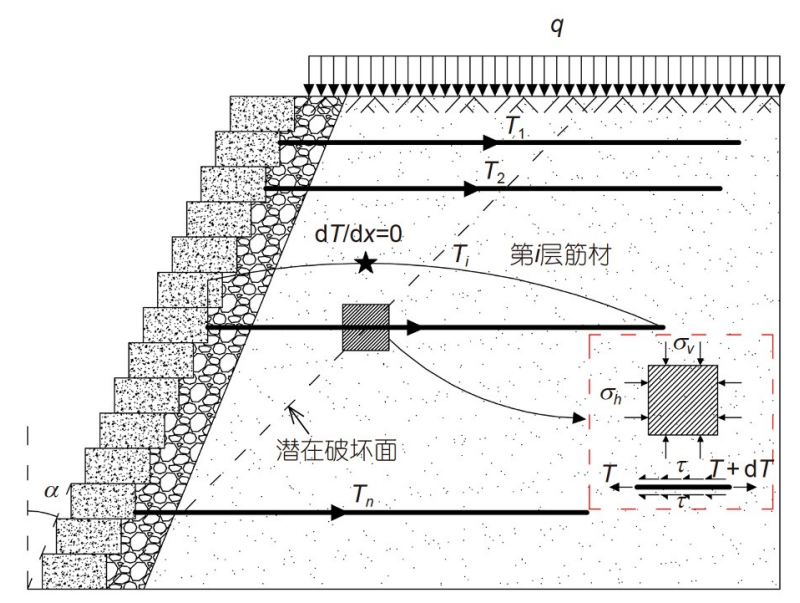

图 1 (网络版彩图)加筋土挡墙结构形式

Figure 1 (Color online) Structural form of geosynthetic-reinforced soil retaining wall.
在简化分析方法方面, 加拿大Bathurst团队 ${ }^{[20-23]}$ 基 于大型试验和现场实测结果, 通过统计回归分析, 提出 了一种估计加筋土挡墙运行期筋材拉力的经验方法 K 刚度法. 一些学者和工程师应用该方法分析了实际工 程的筋材拉力, 一定程度验证了该方法的可行性. 但 是，该方法存在机理不清的问题，也未合理建立筋材 拉力与填土刚度的关系, 对一些工程还存在大大低估 筋材拉力的可能性 ${ }^{[24-27]}$. 考虑筋土协调变形, Ehrlich 和Mitchell ${ }^{[28]}$ 建立了一种分析筋材拉力的非线性弹性 方法, Ehrlich及其团队近十多年来应用该方法开展了 比较广泛的研究, 他们的研究结果表明, 该方法可较 合理地估计直立加筋土挡墙在筋材应变较小条件下的 筋材拉力 ${ }^{[26,27,29]}$. 但是, 该方法未考虑填土的塑性变 形，不能分析倾斜面板的影响，也未量化面板的承载 作用.

以本文作者近 10 年来的研究工作为基础，建立一 套加筋土挡墙在正常工作荷载作用下筋材拉力的塑性 力学分析方法 ${ }^{[30-35]}$. 本文所建立的方法仍基于Ehrlich 和Mitchell ${ }^{[28]}$ 的筋土协调变形原理，但充分考虑填土 的塑性变形, 同时基于力的平衡分析量化面板及其倾 角对筋材拉力的影响.

\section{2 基本假定}

本文所建立的筋材拉力分析方法基于如下假定.

(1) 加筋土挡墙的填土处于平面应变的应力状态, 且加筋土挡墙中正应变为零的方向为中主应力方向;

(2) 土工合成材料筋材水平铺设, 且在运行期正常 工作荷载的作用下, 筋材保持在水平状态;

(3) 在正常工作荷载作用下, 每层筋材的拉力沿长 度方向分布连续、光滑;

（4）在自重及外荷载作用下, 填土的变形主要为塑 性变形, 弹性变形很小, 可以忽略;

(5) 在平面应变条件下, 填土的屈服函数和塑性势 函数受应力罗德角的影响较小, 可以忽略;

(6) 加筋土挡墙墙顶受均布荷载作用, 填土压实带 来的附加筋材拉力可以忽略.

上述假定(1)对大多数加筋土挡墙是符合实际的. 沿轴线方向加筋土挡墙的变形往往很小, 轴线方向的 正应力接近于填土的静止土压力，而沿着筋材拉伸方 向由于填土的变形, 其土压力小于静止土压力. 在施 
工质量有保证的条件下，正常荷载作用下筋材不会出 现应力突变, 因此假定(3)的变形连续、光滑是可以保 证的. 假定(4)对于很多填土也是接近实际情况的. 国 内外的大量单元试验研究结果表明, 很多土材料在变 形非常小的时候已经出现了塑性变形，土材料的小应 变弹性模量较大，其真正意义的弹性可恢复变形很 小 ${ }^{[36,37]}$. 关于假定(6), 填土压实带来的附加筋材拉力, 来自于压实之后填土的超固结变形响应，当挡墙墙顶 的均布荷载比较大时，填土的超固结消失，填土压实 带来的附加筋材拉力也不复存在 ${ }^{[30]}$.

\section{3 筋材内力分析方法推导}

在正常工作荷载作用下，如果筋材层与面板之间 的连接力小于或等于加筋土内部潜在滑移面处的筋材 拉力, 由于筋材层的另一端为自由端, 则根据上述假定 (3), 在加筋土内部潜在滑移面处, 筋材拉力 $T$ 存在如下 关系(如图1所示):

$\frac{\mathrm{d} T}{\mathrm{~d} x}=0$,

其中, $x$ 表征水平方向. 根据该处筋材微段的平衡条件 (如图1所示), 在该处筋材与填土接触面的剪切应力为 零, 因此筋材的拉伸应变与填土的横向应变相同, 筋土 变形协调, 且填土的坚向应力为大主应力 $\sigma_{1}$, 横向应力 为小主应力 $\sigma_{3}$.

在平面应变条件下, 填土的中主应力可表示为

$\sigma_{2}=b\left(\sigma_{1}-\sigma_{3}\right)+\sigma_{3}$,

其中, $b$ 为毕肖普参数. 因而, 填土的平均应力 $p$ 和偏应 力 $q$ 可分别表示为

$p=\frac{\sigma_{1}+\sigma_{2}+\sigma_{3}}{3}=\frac{(1+b) \sigma_{1}+(2-b) \sigma_{3}}{3}$,

$q=\sqrt{3 J_{2}}=\sqrt{b^{2}-b+1}\left(\sigma_{1}-\sigma_{3}\right)$,

而填土的体积应变增量 $\mathrm{d} \varepsilon_{v}$ 和广义剪切应变增量 $\mathrm{d} \varepsilon_{s}$ 可 表示为

$\mathrm{d} \varepsilon_{v}=\mathrm{d} \varepsilon_{1}+\mathrm{d} \varepsilon_{3}$,

$\mathrm{d} \varepsilon_{s}=\sqrt{\frac{4}{3} \mathrm{~d} J^{\prime}{ }_{2}}=\frac{2}{3} \sqrt{\mathrm{d} \varepsilon_{1}^{2}+\mathrm{d} \varepsilon_{3}^{2}-\mathrm{d} \varepsilon_{1} \mathrm{~d} \varepsilon_{3}}$,

其中, $J^{\prime}{ }_{2}$ 为应变张量的第二偏应变不变量. $\varepsilon_{1}$ 为填土的 大主应变, $\varepsilon_{3}$ 为填土的小主应变(压应变为正).
根据上述假定 (5), 填土的屈服函数和塑性势函数 均可表示为 $p$ 和 $q$ 的函数. 这样, 根据上述假定(4)和(5) 以及塑性理论的基本原理, 填土的体积应变增量 $\mathrm{d} \varepsilon_{v}$ 和 广义剪切应变增量 $\mathrm{d} \varepsilon_{s}$ 可表示为

$$
\begin{aligned}
& \mathrm{d} \varepsilon_{v} \cong \mathrm{d} \varepsilon_{v}^{p}=\frac{1}{H}\left(\frac{\partial f}{\partial p} \mathrm{~d} p+\frac{\partial f}{\partial q} \mathrm{~d} q\right) \frac{\partial g}{\partial p}, \\
& \mathrm{~d} \varepsilon_{s} \cong \mathrm{d} \varepsilon_{s}^{p}=\frac{1}{H}\left(\frac{\partial f}{\partial p} \mathrm{~d} p+\frac{\partial f}{\partial q} \mathrm{~d} q\right) \frac{\partial g}{\partial q},
\end{aligned}
$$

其中, $H$ 为填土的塑性模量, $f$ 为填土的屈服函数, $g$ 为塑 性势函数. 式(7b)可进一步表示为

$$
\mathrm{d} \varepsilon_{s} \cong \frac{1}{H}\left(\frac{\partial f}{\partial p} \frac{\mathrm{d} p}{\mathrm{~d} q}+\frac{\partial f}{\partial q}\right) \frac{\partial g}{\partial q} \mathrm{~d} q,
$$

在填土的塑性势面上，塑性势函数的全微分 $\mathrm{d} g=0$, 因此,

$\mathrm{d} g=\frac{\partial g}{\partial p} \mathrm{~d} p+\frac{\partial g}{\partial q} \mathrm{~d} q=0$,

也就是说,

$\frac{\mathrm{d} p}{\mathrm{~d} q}=-\frac{\partial g}{\partial q} / \frac{\partial g}{\partial p}$,

将式(9)代入式(8)中, 填土的应力应变关系可以表示为

$\mathrm{d} \varepsilon_{s} \cong \frac{1}{H}\left[-\frac{\frac{\partial f}{\partial p}\left(\frac{\partial g}{\partial q}\right)^{2}}{\frac{\partial g}{\partial p}}+\frac{\partial f}{\partial q} \frac{\partial g}{\partial q}\right] \mathrm{d} q$,

令 $\mathrm{d} \varepsilon_{3}=-\beta \mathrm{d} \varepsilon_{1}$, 将式(4)和(6)代入式(10), 可得

$\mathrm{d} \sigma_{1}-\mathrm{d} \sigma_{3} \cong D_{t} \mathrm{~d} \varepsilon_{1}$,

$$
D_{t}=\frac{2 H \sqrt{1+\beta+\beta^{2}}}{3\left[-\frac{\frac{\partial f}{\partial p}\left(\frac{\partial g}{\partial q}\right)^{2}}{\frac{\partial g}{\partial p}}+\frac{\partial f}{\partial q} \frac{\partial g}{\partial q}\right] \sqrt{b^{2}-b+1}},
$$

$D_{t}$ 可理解为平面应变条件下应力应变关系曲线的斜 率. 可以看出, $D_{t}$ 是由应力状态、填土塑性模量以及填 土的剪胀性共同决定. 式(11)中, $\beta$ 与填土的剪胀性质 有关, 决定于填土的应力-剪胀关系. $\beta>0$, 且当填土体 积剪胀时, $\beta<1$, 剪缩时, $\beta>1$.

现在加筋土的内部潜在滑移面上, 筋土变形协调, 因此, $\left|\mathrm{d} \varepsilon_{3}\right|=\mathrm{d} \varepsilon_{r}$, 其中 $\varepsilon_{r}$ 为筋材拉伸应变, 为正值. 在加 
筋土挡墙中，若忽略面板对潜在滑移面处筋材拉力的 影响, 填土的横向土压力与筋材拉力平衡, 则

$\mathrm{d} \sigma_{3}=\frac{\mathrm{d} T}{f\left(S_{v}\right)}=-\frac{J_{t} \mathrm{~d} \varepsilon_{3}}{f\left(S_{v}\right)}$,

式中, $J_{t}$ 为筋材在服役期的切线刚度, $f\left(S_{v}\right)$ 为筋材坚向 间距 $S_{v}$ 的函数. 以往大量的研究结果表明, 当加筋土挡 墙为直立墙时 (图 1 中 $\alpha=0$ ), $f\left(S_{v}\right)=S_{v}$, 但当面板倾斜时 $(\alpha>0), f\left(S_{v}\right) \neq S_{v}$. 这样式(11a) 可改写为

$\mathrm{d} \sigma_{1}+\frac{J_{t} \mathrm{~d} \varepsilon_{3}}{f\left(S_{v}\right)} \cong-\frac{D_{t}}{\beta} \mathrm{d} \varepsilon_{3}$,

$\mathrm{d} \varepsilon_{3} \cong-\frac{f\left(S_{v}\right) \beta}{\beta J_{t}+f\left(S_{v}\right) D_{t}} \mathrm{~d} \sigma_{1}$,

因此, 筋材的拉力增量 $\nabla T$ 为

$\nabla T=\frac{J_{t} f\left(S_{v}\right) \beta}{\beta J_{t}+f\left(S_{v}\right) D_{t}} \nabla \sigma_{1}$,

式中, $\nabla \sigma_{1}$ 为筋材层在内部潜在滑移面处的坚向应力 增量. 当坚向应力的增量非常小时, 横向应力和坚向 应力的比值可表示为

$K_{r}=\frac{\sigma_{3}}{\sigma_{1}} \cong \frac{\sigma_{3}+\nabla \sigma_{3}}{\sigma_{1}+\nabla \sigma_{1}} \cong \frac{\sigma_{3}+K_{r} \nabla \sigma_{1}}{\sigma_{1}+\nabla \sigma_{1}}$,

式(15)合理性的解释参见文献[34]. 这样,

$\nabla T=f\left(S_{v}\right) \nabla \sigma_{3}=K_{r} f\left(S_{v}\right) \nabla \sigma_{1}$,

将式(16)代入式(14), 可得

$K_{r}=\frac{J_{t} \beta}{\beta J_{t}+f\left(S_{v}\right) D_{t}}$,

给定填土的应力应变关系及应力剪胀方程, 给定函数 $f\left(S_{v}\right)$, 式(17)即可用于计算加筋土的侧向土压力系数, 而筋材的拉力可由式(18)得到

$T_{0}=K_{r} f\left(S_{v}\right) \sigma_{1}=\frac{f\left(S_{v}\right) J_{t} \beta}{\beta J_{t}+f\left(S_{v}\right) D_{t}} \sigma_{1}$,

式中, $\sigma_{1}$ 为当前筋材层处的坚向土压力.

平面应变状态下式(11)填土的应力应变关系可用 不同形式的应力状态函数给出 ${ }^{[38-40]}$. 国内外学者的一 些研究结果表明，土材料的平面应变压缩应力应变关 系曲线在峰值强度之前可近似采用双曲线方程描 述 $^{[41-44]}$, 则根据邓肯张双曲线应力应变关系模型 ${ }^{[45]}$,

$D_{t}=k p_{a}\left(\frac{\sigma_{3}}{p_{a}}\right)^{n}\left[1-R_{f} \frac{\left(\sigma_{1}-\sigma_{3}\right)(1-\sin \phi)}{2 c \cos \phi+2 \sigma_{3} \sin \phi}\right]^{2}$,
其中, $c$ 和 $\phi$ 为填土的平面应变强度参数, $R_{f}$ 为破坏比, $k$ 和 $n$ 为曲线拟合参数. 由于 $\sigma_{3}=K_{r} \sigma_{1}$, 式(19a)可改写为

$D_{t}=k p_{a}\left(\frac{K_{r} \sigma_{1}}{p_{a}}\right)^{n}\left[1-R_{f} \frac{\sigma_{1}\left(1-K_{r}\right)(1-\sin \phi)}{2 c \cos \phi+2 K_{r} \sigma_{\mathrm{s}} \sin \phi}\right]^{2}$.

在平面应变条件下, 填土的剪胀比为

$\mathrm{d} g=\frac{\mathrm{d} \varepsilon_{v}^{p}}{\mathrm{~d} \varepsilon_{s}^{p}} \cong \frac{\mathrm{d} \varepsilon_{v}}{\mathrm{~d} \varepsilon_{s}}=\frac{3(1-\beta)}{2 \sqrt{1+\beta+\beta^{2}}}$,

因此, 给定 $\mathrm{d} g$ 与填土应力状态的关系, $\beta$ 与应力状态的 关系即可确定. 若填土的剪胀可用Rowe剪胀理论进行 描述, 则

$\mathrm{d} \varepsilon_{1}=-K \frac{\sigma_{3}}{\sigma_{1}} \mathrm{~d} \varepsilon_{3}=-K K_{r} \mathrm{~d} \varepsilon_{3}$,

其中, $K$ 为Rowe剪胀系数, 可用式(22)计算,

$K=\frac{1+\sin \phi}{1-\sin \phi} \frac{1-\sin \psi}{1+\sin \psi}$,

其中, $\psi$ 为剪胀角. 因此 $\beta$ 可表示为

$\beta=\frac{1}{K K_{r}}$.

\section{4 直立加筋挡墙筋材内力分析}

忽略面板对筋材拉力的影响, 对于直立加筋挡墙,

$\mathrm{d} \sigma_{3}=\frac{\mathrm{d} T}{S_{v}}=-\frac{J_{t} \mathrm{~d} \varepsilon_{3}}{S_{v}}$,

应用Rowe剪胀理论和式(23), 则式(17)可改写为

$K_{r}=\frac{J_{t} / S_{v}}{K K_{r} D_{t}+J_{t} / S_{v}}$,

式(25)为 $K_{r}$ 的隐函数，可应用迭代的方法求解 $K_{r}$. 求得 $K_{r}$ 之后, 给定筋材层位置处填土的坚向土压力 $\sigma_{1}$, 则筋 材在潜在滑移面处的拉力为

$T_{0}=\frac{J_{t} \sigma_{1}}{K K_{t} D_{t}+J_{t} / S_{v}}$.

\section{5 倾斜面板加筋挡墙筋材拉力分析}

面板的倾角对潜在滑移面处筋材拉力的大小是有 影响的. 在填土、筋材、墙高、面板形式、挡墙荷载 完全相同的条件下, 随着面板倾角的增大(如图1所示), 筋材拉力逐渐减小. 在国内外的很多设计规范中 ${ }^{[5 \sim 8]}$, 
倾角 $\alpha \leq 20^{\circ}$ 的加筋土结构归类为挡墙，当 $\alpha>20^{\circ}$ 时，一 般按加筋土边坡进行设计. 本文的研究对象为加筋土 挡墙, 因此墙面最大倾角约为 $20^{\circ}$. 国内外的大多数设 计规程建议, 当 $\alpha>10^{\circ}$ 时, 在强度极限条件下倾角对筋 材拉力的影响可以采用库仑土压力的水平分量予以考 虑. 但是, 在正常工作荷载作用下, 填土的强度往往未 完全发挥，而库仑土压力理论采用填土的摩擦角计算 土压力系数. 此外, 加拿大加筋土专家Bathurst团队基 于监测结果，提出了一种基于统计分析的面板倾角影 响计算方法，并将其应用于该团队提出的 $\mathrm{K}$ 刚度法中. 应用该方法得到的筋材拉力随着面板倾角的增大而降 低, 但降低的幅度不大.

从式(18)可以看出，面板倾角的影响可以通过函 数 $f\left(S_{v}\right)$ 和填土的坚向土压力 $\sigma_{1}$ 进行量化. 在加筋土挡 墙潜在滑移面附近，填土的坚向土压力受面板倾角的 影响. 图 2 为有限元分析的结果, 其中 $\alpha=21^{\circ}$, 在潜在滑 移面位置, 下层筋材上的坚向土压力较 $\gamma z+q$ 小. 此外, 基于土力学理论推导函数 $f\left(S_{v}\right)$ 的具体形式是比较困难 的. 基于上述考虑, 本文应用力学平衡分析思路, 推导 倾斜面板对筋材内力的影响, 并将其与第4节的筋材拉 力分析方法相结合，计算倾斜面板加筋土挡墙的筋材 拉力.

现行的大多数设计规程采用库仑土压力水平分量 考虑倾角对筋材拉力的影响 ${ }^{[5-8]}$, 传统库仑土压力理论 基于楔形体受力平衡分析，作用在支挡结构上的土压 力方向取决于墙背与填土的摩擦角, 这与加筋土挡墙 存在一定区别. 在不考虑面板对筋材内力影响的条件 下，倾斜面板加筋土挡墙在正常工作荷载作用下，筋

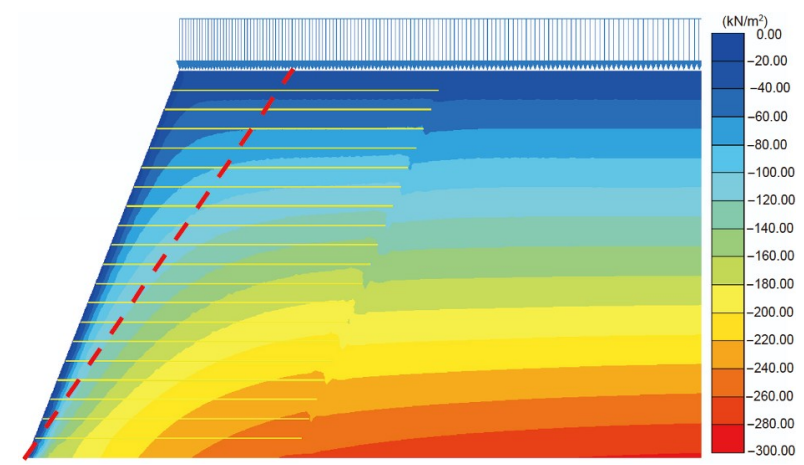

图 2 (网络版彩图)正常工作荷载作用下加筋土挡墙坚向应 力云图

Figure 2 (Color online) Vertical stress nephogram of GRS retaining wall under working load.
材拉力保持水平状态，图3为其受力平衡分析简图. 图 中 $E_{\alpha}$ 为考虑倾角影响的加筋等效土压力, $W$ 为楔形土 体自重, $V$ 为附加荷载, 由墙顶均布荷载 $q$ 计算, $F$ 为楔 形土体与背后土体之间的作用力，其作用方向 $\phi_{\mathrm{mob}}$ 由 填土发挥强度决定, $\theta$ 为潜在滑动面倾角. 依照力的平 衡分析，可得到考虑倾角影响的加筋土挡墙土压力计 算公式:

$E_{\alpha}=(W+V) \tan \left(\theta-\phi_{\mathrm{mob}}\right)$,

$E_{\alpha}=\left(\frac{\gamma H^{2}}{2}+q H\right) K_{\alpha}$

$K_{\alpha}=$

$\frac{\sin \phi_{\mathrm{mob}} \sin \left(\alpha+\phi_{\mathrm{mob}}\right)+\cos \alpha-2 \sqrt{\sin \phi_{\mathrm{mob}} \cos \alpha \sin \left(\alpha+\phi_{\mathrm{mob}}\right)}}{\cos \alpha \cos ^{2} \phi_{\mathrm{mob}}}$,

其中, $K_{\alpha}$ 为考虑倾角影响的加筋土挡墙土压力系数, 在 $\alpha=0^{\circ}$ 时, $K_{\alpha=0}$ 与朗肯土压力系数的表达式一致. $\phi_{\mathrm{mob}}$ 为 工作荷载作用下填土发挥强度对应的摩擦角. 倾斜面 板加筋土挡墙的填土实际发挥强度较难获取，本文近 似以直立挡墙的填土发挥强度代替:

$\phi_{\mathrm{mob}}=90^{\circ}-2 \arctan \left(\sqrt{K_{r}}\right)$,

其中， $K_{r}$ 为第 4 节中直立加筋土挡墙的侧向土压力系 数. 这样, 将力学平衡思路与筋材拉力分析方法相结 合, 可得到倾斜面板加筋挡墙筋材拉力计算公式如下:

$T_{\alpha}=T_{0} \frac{K_{\alpha}}{K_{\alpha=0}}$.

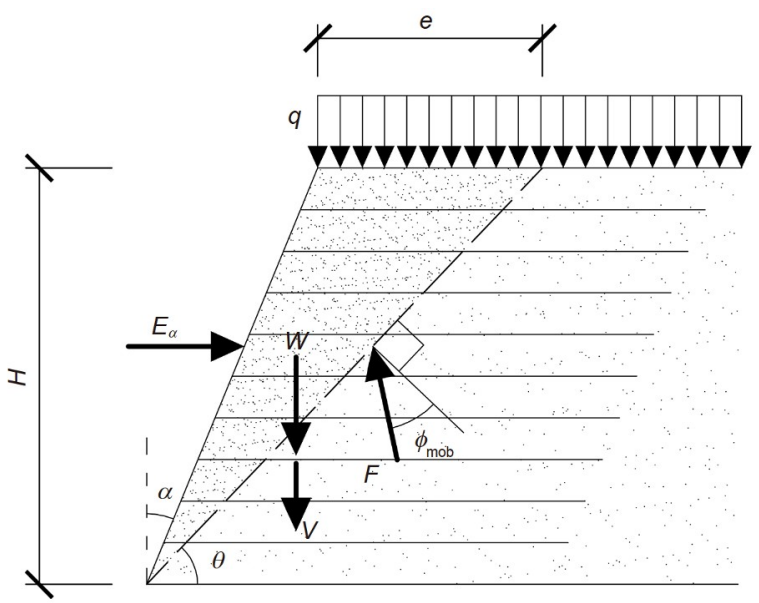

图 3 倾斜面板加筋挡墙受力分析

Figure 3 Force equilibrium of GRS retaining wall with inclined facing. 


\section{6 模块式面板对筋材拉力的影响分析}

国内外主流的加筋土挡墙设计方法进行加筋土挡 墙的内部稳定校核时，一般不考虑面板对内部稳定的 整体贡献，面板的作用主要是保证面板附近处填土的 局部稳定. 但是, 在正常工作荷载作用下, 面板发挥了 一定的承载作用. 国内外大量的大型试验和现场监测 结果表明，正常工作荷载作用下筋材的拉力都不大， 特别是, 筋材层的拉力在挡土墙的中下部往往不会随 着上覆土层厚度或坚向土压力的增大而增大.

筋材拉力的这种变化特点源于面板底部的约束. 如图4所示, 以面板为分析对象, 当面板底部无约束时, 面板-筋材连接力与作用于面板的土压力相互平衡. 这 时面板对潜在滑移面处的筋材拉力基本没有影响，主 动区填土的稳定由潜在滑移面处土的剪切抗力以及筋 材拉力共同承担. 但是当面板的底部受地基土约束时, 作用于面板的横向土压力将部分由面板底部的横向约 束承担, 面板的土压力与筋材-面板连接力不再相互平 衡, 同时, 面板的底部约束对主动区填土的稳定也做出 了贡献, 筋材的拉力随着降低. 但是, 面板底部约束的 影响主要集中于挡墙下部, 随着高度的上升, 面板约束

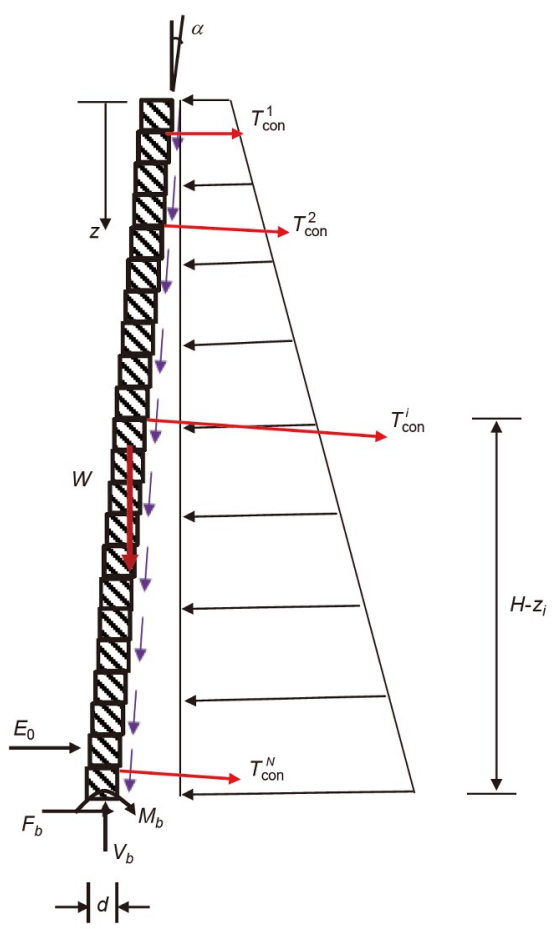

图 4 (网络版彩图)模块式面板受力简图 Figure 4 (Color online) Force equilibrium of modular block facing.
的影响逐渐较小，在挡墙的中部和上部，筋材-面板连 接力与土压力相互平衡, 面板对潜在滑移面处筋材拉 力的影响也就消失了. 上述加筋土挡墙的承载机理亦 可以用加筋土的横向变形进行解释. 面板可以视为底 部约束、受土压力和筋材-面板连接力作用的悬臂梁. 在靠近面板底部的部位, 面板对加筋土变形的约束较 大，这时填土和筋材的变形相对较小，筋材拉力自然 也不大. 随着高度的上升, 面板悬臂梁的这种变形约 束作用逐渐减小，填土和筋材的协调变形关系恢复到 式(17)的状态.

基于上述分析，本文采用如下思路考虑面板对筋 材拉力的影响: 首先, 通过对面板的平衡分析, 量化面 板底部的横向约束力; 其次, 针对每层筋材和填土, 通 过分析其平衡量化面板约束对筋材拉力的影响. 如图4 所示, 面板的土压力可应用库仑土压力理论进行分析:

$$
\begin{aligned}
& E_{a}=E_{a 1}+E_{a 2}, \\
& E_{a 1}=K_{a} \frac{1}{2} \gamma H^{2}, \\
& E_{a 2}=K_{a} q H,
\end{aligned}
$$

其中, $K_{a}$ 为库仑土压力系数:

$$
K_{a}=\frac{\cos ^{2}(\varphi+\alpha)}{\cos ^{2} \alpha \cos (\delta-\alpha)\left[1+\sqrt{\frac{\sin (\delta+\varphi) \sin \varphi}{\cos (\delta-\alpha) \cos \alpha}}\right]^{2}} .
$$

忽略筋材-面板连接力的坚向分量, 则面板坚向力 的平衡方程和面板横向力的平衡方程为

$$
\begin{aligned}
& W+E_{a} \sin (\delta-\alpha)=V_{b}, \\
& F_{b}=E_{a} \cos (\delta-\alpha)-E_{0}-\sum T_{\text {con }}^{i h} \leq V_{b} \tan \delta_{b},
\end{aligned}
$$

式中, $T_{\mathrm{con}}^{i h}$ 筋材-面板连接力的水平分量, 而 $\delta_{b}$ 为面板底 部与地基土接触的摩擦角. 以面板底部中心为力矩中 心, 面板的力矩平衡方程可以表示为

$$
\begin{aligned}
\sum T_{\mathrm{con}}^{i h}\left(H-z_{i}\right)+E_{a 1} \sin (\delta-\alpha)\left(\frac{d}{2}+\frac{H}{3} \tan \alpha\right) \\
+E_{a 2} \sin (\delta-\alpha)\left(\frac{d}{2}+\frac{H}{2} \tan \alpha\right) \\
=E_{a 1} \cos (\delta-\alpha) \frac{H}{3}+E_{a 2} \cos (\delta-\alpha) \frac{H}{2}-M_{b} \\
-E_{0} h_{0}-W \frac{H}{2} \tan \alpha,
\end{aligned}
$$

式中, $d$ 是面板的宽度, $z_{i}$ 第 $i$ 层筋材的深度, $h_{0}$ 为面板前 
土压力 $E_{0}$ 的作用点高程, $M_{b}$ 为面板底部的反力矩, 假 定面板与基地无脱开, 则 $M_{b}$ 的最小值为零, 最大值为 $M_{b}=V_{b} d / 6$. 基于上述公式, 可以求得筋材连接带来的 抗力矩 $\sum T_{\mathrm{con}}^{i \text { h }}\left(H-z_{i}\right)$.

但是, 为了求得筋材-面板连接力的分布, 需要假 定如图5所示的筋材水平连接力分布图. 这里 $\mathrm{B}$ 点由 式(36)和(37)共同决定, 并非定值. B点以上, 筋材-面 板连接力与作用于面板的土压力相互平衡, 在 $\mathrm{B}$ 点以下, 土压力大于连接力. 筋材-面板水平连接力的分布以文 献中的一些实测和数值分析结果为基础, 如图 5 所示.

获得面板基底反力和筋材-面板连接力之后, $\mathrm{B}$ 点 以下面板约束对潜在滑移面处筋材拉力的影响可以用 图6的简图进行分析. 即:

$T_{f}=p_{h} S_{v}-T_{\mathrm{con}}^{i h}=K_{a h} \sigma_{\mathrm{P}} S_{v}-T_{\mathrm{con}}^{i h}$,

式中, $p_{h}$ 为每层加筋土(如图6所示)位置作用于面板横 向土压力的平均值, $K_{a h}$ 为库仑土压力系数的水平分 量. 这样, B点以下潜在滑移面处筋材的拉力可以结合 式(18)和(38)获得, 即:

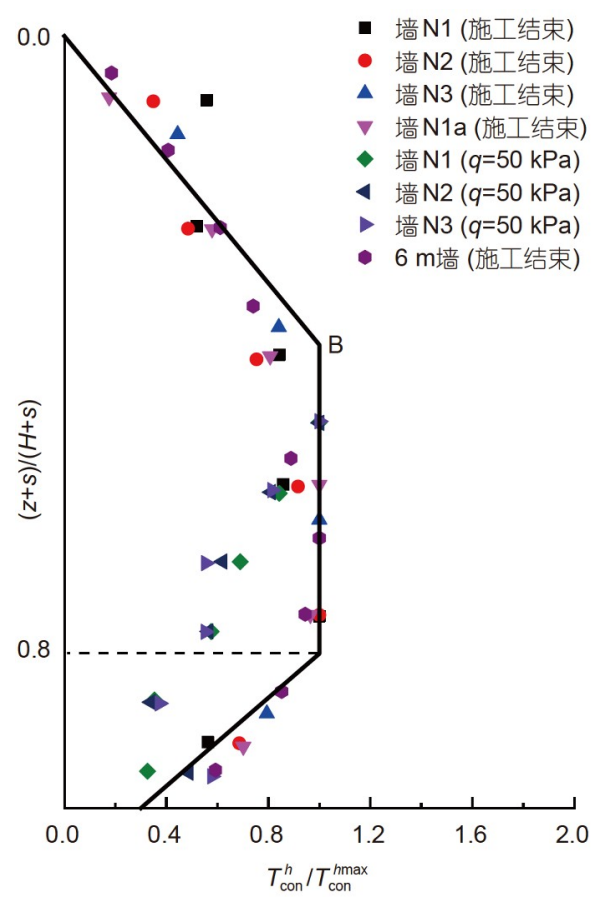

图 5 (网络版彩图)筋材-面板水平连接力分布图(离散点为 文献 $[41,42,46]$ 的数值分析结果)

Figure 5 (Color online) Reinforcement-facing horizontal connection load distribution (discrete points are the numerical analysis results from refs. $[41,42,46])$.
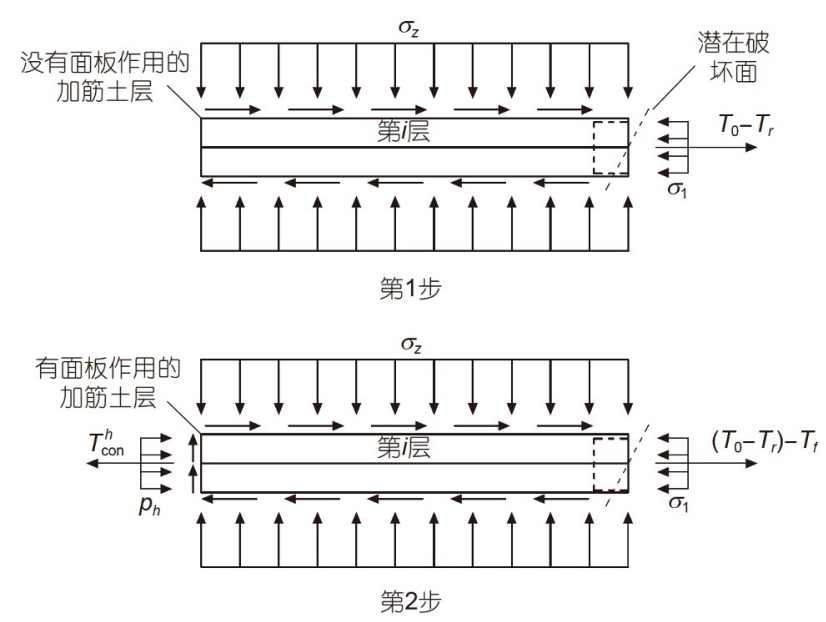

图 6 面板对潜在破坏面位置筋材拉力的影响分析简图

Figure 6 The influence of the facing on the reinforcement load at potential failure surface.

$T=T_{\alpha}-T_{f}$.

需要强调的是, 在 $\mathrm{B}$ 点以上, 面板的约束作用消失, $T=T_{\alpha}$.

\section{7 分析方法验证}

本文应用文献中的试验实测结果和本文的有限元 分析结果验证上述分析方法的有效性. 为方便描述, 本 文将上述分析方法取名为塑性力学分析法. 本节的验 证依据如下思路展开. 首先, 开展一系列非线性有限元分 析，计算不同面层倾角条件下返包式面层加筋土挡墙 的筋材拉力. 返包式面层的刚度很小, 其对加筋土挡 墙筋材拉力的影响很小, 可以忽略不计, 这些有限元 结果因而可以验证无面板影响条件下筋材拉力塑性力 学分析法的可靠性. 之后, 应用文献中的大型模型试验 结果和数值模拟结果，验证本文提出的模块式面板基 底约束反力估算方法的合理性. 最后, 针对文献中三 组实际加筋土挡墙展开分析, 通过比较实测和估算的 筋材拉力证明本文方法对实际加筋土结构的有效性.

\section{1 返包式面层加筋土挡墙有限元分析}

本文的加筋土挡墙有限元分析模型与 Wang等 人 $^{[47]}$ 的模型相同. 该模型的可靠性在该论文中有详细 阐述, 本文因篇幅关系不予赘述. 该模型应用Plaxis2D 建立, 填土采用硬土模型模拟, 返包式面层采用刚度很 
小的梁单元模拟，而筋材模型为一维的土工格栅单元, 材料性质为弹性. 在有限元分析中, 采用等效压力模拟 施工过程中的填土压实作用 ${ }^{[29,48]}$.

本文分析的模型加筋土挡墙高 $8 \mathrm{~m}$ ，包括5种面板 倾角，其中一种倾角有限元模型的网格如图7所示. 加 筋土挡墙的填土为密实砂土，其模型参数来自于文献 [46], 如表1所示. 在挡墙顶部, 施加了 $20 \mathrm{kPa}$ 的均布荷 载. 加筋土挡墙的筋材长 $7 \mathrm{~m}$, 加筋间距为 $0.5 \mathrm{~m}$, 筋材 刚度 $J=700 \mathrm{kN} / \mathrm{m}$. 图8给出加筋土挡墙的筋材拉力沿高 程的分布.

根据硬土模型参数的物理意义，可以推导得到式 (19)中的模型参数与硬土模型参数的关系, 从而获得 塑性力学方法所需的部分填土参数, 如表 1 所示. 模型 加筋土挡墙的其他参数, 包括填土强度、剪胀角、筋 材刚度等与有限元模型相同. 图8给出了不同面板倾 角条件下，应用本文提出的分析方法得到的筋材拉力 与有限元分析得到筋材拉力的比较. 可以看出, 两者 之间的差别较小，本文的方法具备足够的精度. 图中 同时给出了两种倾角条件下美国FHWA规范的筋材拉 力计算结果. 可以看出, 基于FHWA规范, 在无面板作 用的前提下, 筋材拉力偏小很多.

\section{2 面板底部约束力分析方法的验证}

Huang等人 ${ }^{[49]}$ 开展了一系列的大型模型试验，并 在模型试验中测量了挡墙底部的横向和坚向反力. 试 验的加筋土挡墙模板为混凝土模块. 本节应用这些试 验结果验证式(32) (37)的合理性. 这里, 所用到的参 数包括砂性填土的摩擦角、面板的底宽以及面板的倾 角. 这些参数均直接来自于Bathurst等的论文或报 告 ${ }^{[21,22,49 \sim 51]}$, 参见表2. 图9比较了试验得到面板底部反 力与本方法的估算反力. 可以看出, 两者的差别是可以 接受的. 由于本文的方法忽略了筋材对面板的坚向连 接力, 因此本文得到的结果较试验得到结果稍小.

Chen等人 ${ }^{[50]}$ 开展了一系列的有限差分数值分析, 研究了面板底部约束对筋材拉力的影响, 模型参数参 见表2. 图10给出了Chen等人 ${ }^{[50]}$ 得到的面板基底反力 与式(36)估算结果的比较. 可以看出, 两者之间的差别 比较小.

\section{3 实测结果与塑性力学分析结果比较}

Nelson ${ }^{[51]}$ 开展了一组大型模型试验, 墙高 $3.6 \mathrm{~m}$,

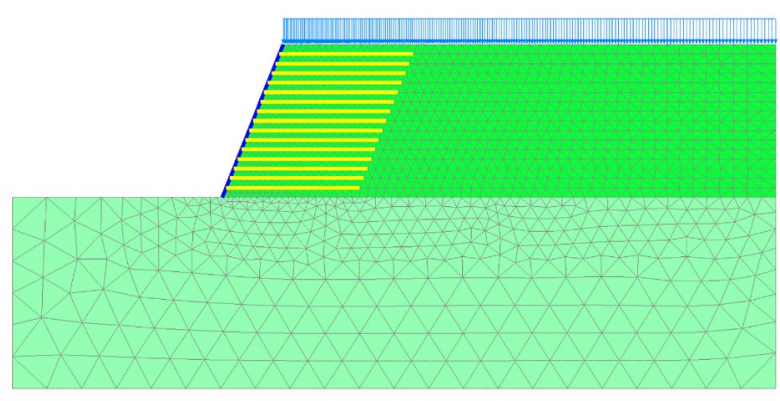

图 7 (网络版彩图)返包式倾斜加筋挡墙有限元模型网格 Figure 7 (Color online) The FEM mesh of the wrapped GRS retaining wall with inclined facing.

表 1 加筋土挡墙填土参数

Table 1 Filling parameters of GRS retaining wall

\begin{tabular}{cc||cc}
\hline 参数 & 数值 & 参数 & 数值 \\
\hline$\gamma\left(\mathrm{kN} / \mathrm{m}^{3}\right)$ & 17.1 & $n$ & 0.5 \\
$c(\mathrm{kPa})$ & 0.3 & $k_{b}$ & 157.5 \\
$\phi\left(^{\circ}\right)$ & 41.0 & $m$ & 0.5 \\
$\psi\left({ }^{\circ}\right)$ & 11.0 & $E_{50}^{\mathrm{ref}}\left(\mathrm{kN} / \mathrm{m}^{2}\right)$ & 28000.0 \\
$R_{f}$ & 0.9 & $E_{\text {oed }}^{\mathrm{ref}}\left(\mathrm{kN} / \mathrm{m}^{2}\right)$ & 28000.0 \\
$k$ & 509.0 & $E_{u r}^{\mathrm{ref}}\left(\mathrm{kN} / \mathrm{m}^{2}\right)$ & 72500.0 \\
$k_{u r}$ & 725.0 & - & - \\
\hline
\end{tabular}

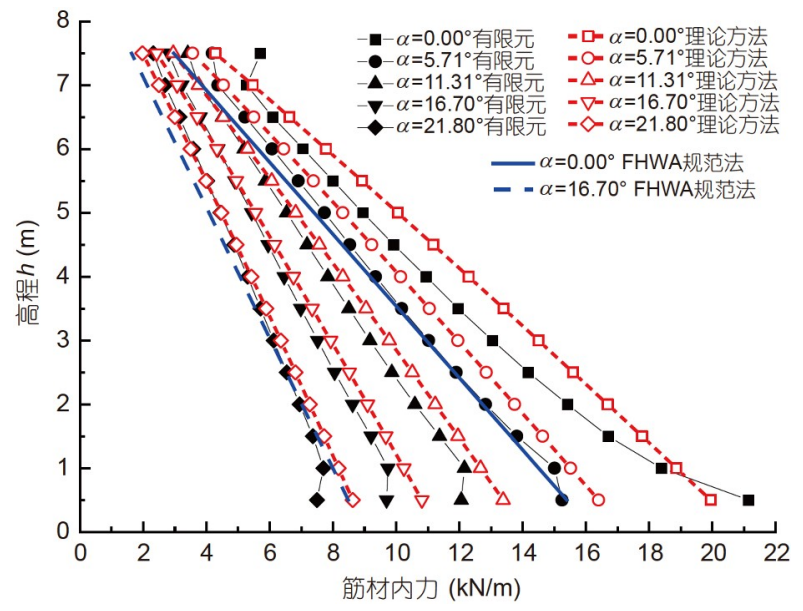

图 8 (网络版彩图)返包式倾斜加筋挡墙筋材拉力分布

Figure 8 (Color online) The reinforcement loads distribution of the wrapped GRS retaining wall with inclined facing.

该加筋土挡墙修筑于刚性地基上，采用 $0.14 \mathrm{~m}$ 厚度的 砌块式面板, 并在最底部砌块上安装了水平方向的弹 
簧限制其水平滑动, 加筋土挡墙筋材坚向间距为 $0.6 \mathrm{~m}$,

其筋材刚度可采用切线刚度描述:

$$
J_{t}=1 /\left[115\left(1 / 115+0.11 \varepsilon_{r}\right)^{2}\right] \text {, }
$$

其中, $\varepsilon_{r}$ 为筋材应变. 其他模型参数参见表 2 . 挡墙顶部

表 2 文献中加筋土挡墙填土参数

Table 2 Backfill parameters of GRS retaining walls in the literature

\begin{tabular}{|c|c|c|c|c|c|}
\hline & 参数 & RMC足尺试验 & Chen有限差分模拟 & Allen和Bathurst足尺试验 & Nelson大型模型试验 \\
\hline \multirow{11}{*}{ 填土 } & $\gamma\left(\mathrm{kN} / \mathrm{m}^{3}\right)$ & 16.8 & 22 & 21.7 & 16.4 \\
\hline & $c(\mathrm{kPa})$ & - & - & 2 & 2 \\
\hline & $\phi\left(^{\circ}\right)$ & 44 & 48 & 54 & 44 \\
\hline & $\delta\left({ }^{\circ}\right)$ & 44 & 48 & 43 & 44 \\
\hline & $\psi\left({ }^{\circ}\right)$ & 11 & 11 & 20 & 11 \\
\hline & $k$ & 1968 & 1968 & 1800 & 1968 \\
\hline & $n$ & 0.5 & 0.5 & 0.6 & 0.5 \\
\hline & $R_{f}$ & 0.86 & 0.86 & 0.85 & 0.86 \\
\hline & $B_{i}$ & 27000 & 27000 & 7500 & 27000 \\
\hline & $k_{u r}$ & 2362 & 2362 & 2160 & 2362 \\
\hline & $\mu_{u r}$ & 0.234 & 0.234 & 0.16 & 0.234 \\
\hline \multirow{5}{*}{ 面板 } & $\gamma\left(\mathrm{kN} / \mathrm{m}^{3}\right)$ & 23 & 23 & 21 & 23 \\
\hline & $d(\mathrm{~m})$ & 0.3 & 0.3 & 0.3 & 0.14 \\
\hline & $E_{0}(\mathrm{kN} / \mathrm{m})$ & 0 & 0 & $4.56 / 3.32$ & 0 \\
\hline & $h_{0}(\mathrm{~m})$ & - & - & $0.367 / 0.267$ & - \\
\hline & $M_{b}(\mathrm{kN} \mathrm{m})$ & $V_{b} d / 6$ & - & $V_{b} d / 6$ & $V_{b} d / 6$ \\
\hline \multirow{3}{*}{ 加筋土挡墙 } & $H(\mathrm{~m})$ & 3.6 & $3.6-12$ & $6.3 / 10.7$ & 3.6 \\
\hline & $S_{v}(\mathrm{~m})$ & $\begin{array}{c}\text { 0.6-墙 } 1,2,5,10 \\
\text { 0.9-墙3 }\end{array}$ & 0.6 & 0.6 & 0.6 \\
\hline & $\alpha\left(^{\circ}\right)$ & $\begin{array}{c}\text { 8-墙 } 1,2,3,5 \\
\text { 13-墙 } 10\end{array}$ & 8 & 0 & 13 \\
\hline
\end{tabular}
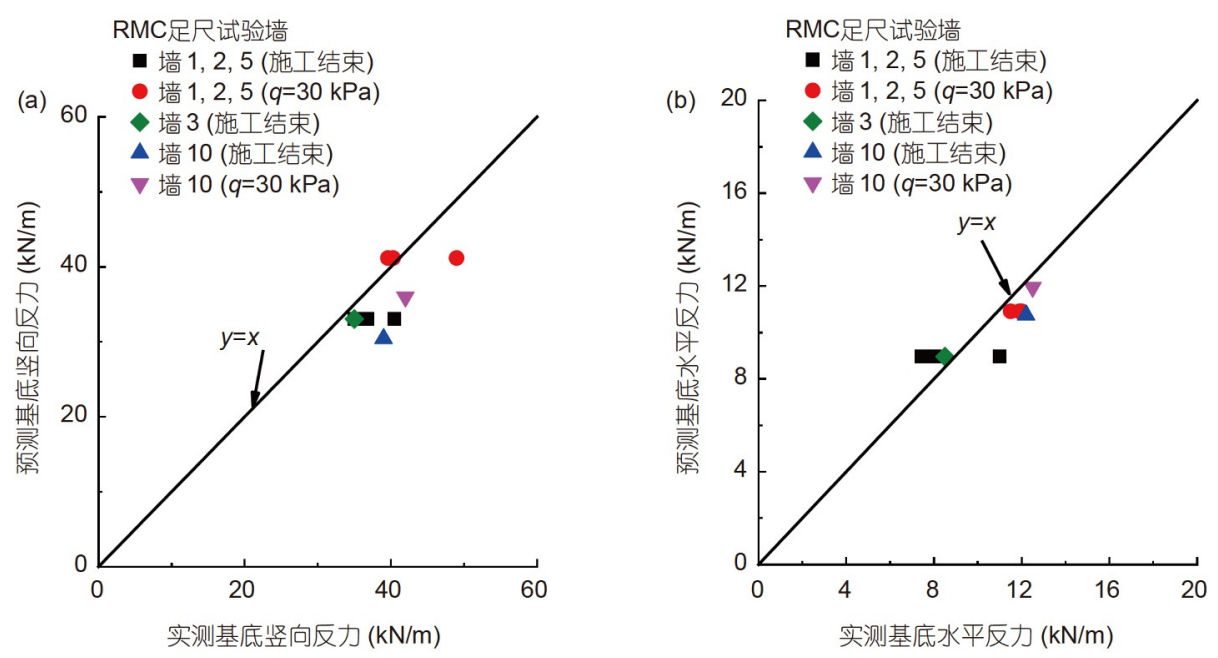

图 9 (网络版彩图)模块式面板基底反力实测结果与分析结果比较. (a) 坚向反力; (b) 水平反力

Figure 9 (Color online) Comparison of the measured and predicted results of the toe resistances. (a) Vertical toe resistances; (b) horizontal toe resistances. 


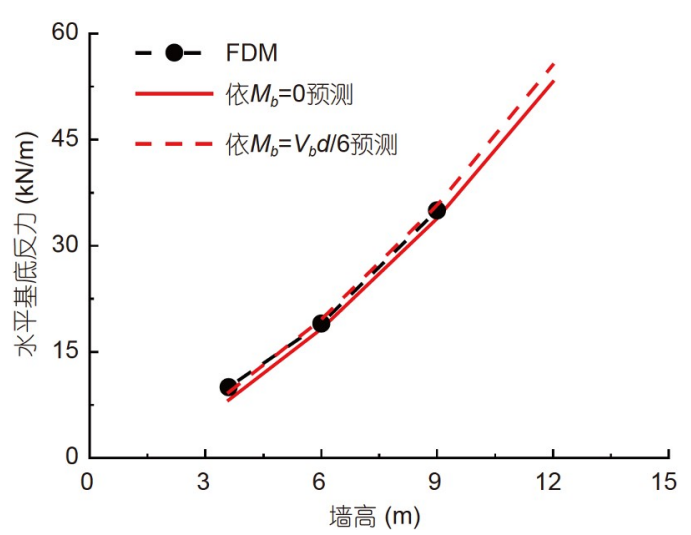

图 10 (网络版彩图)模块式面板基底水平反力FDM数值模 拟结果与分析结果比较

Figure 10 (Color online) Comparison of the predicted and FDM analyzed results of the toe resistances.

施加了 $10 \mathrm{kPa}$ 的均布压力. 图11给出了试验实测筋材 拉力和塑性分析方法估算拉力的比较. 可以看出, 本 文的方法能够较准确地估算加筋土挡墙在正常工作荷 载作用下的筋材拉力.

Allen和Bathurst ${ }^{[21,22]}$ 介绍了两组模块式加筋土挡 墙的实测结果, 墙高分别为 6.3 和 $10.7 \mathrm{~m}$. 本文应用上述 的塑性力学筋材拉力分析方法估计这两组挡墙的筋材 拉力. $\mathrm{Yu}$ 等人 ${ }^{[52]}$ 应用室内试验确定了这两组挡墙填土 及筋材的力学性能, 并应用有限差分法分析了这两组 挡墙的响应. 本文塑性力学分析方法的模型参数均来 自于该文献，参见表2. 如图12所示，本文方法对潜在 滑移面附近筋材拉力的估计较实测值稍大，但两者的 差别在可以接受的范围之内.

\section{8 讨论与结论}

基于塑性力学基本理论和土工合成材料加筋土挡 墙筋土协调变形基本原理, 结合合理的假定, 本文推导 了正常工作荷载作用及无面板基底约束条件下的加筋 土挡墙筋材拉力分析方法, 并在其基础上, 基于模块式 面板的静力平衡，量化了面板基底约束对筋材拉力的 影响. 本文所提出的方法, 可应用于分析土工合成材 料加筋土挡墙在服役期的筋材拉力, 为挡墙结构的服 役极限状态分析提供一种工具.

本文所提出的方法仅适用于倾角较小的加筋土挡 墙, 对于倾角较大的加筋土边坡并不适用. 本文的方法 仅考虑了返包式和模块式两种面板形式，对于连续现

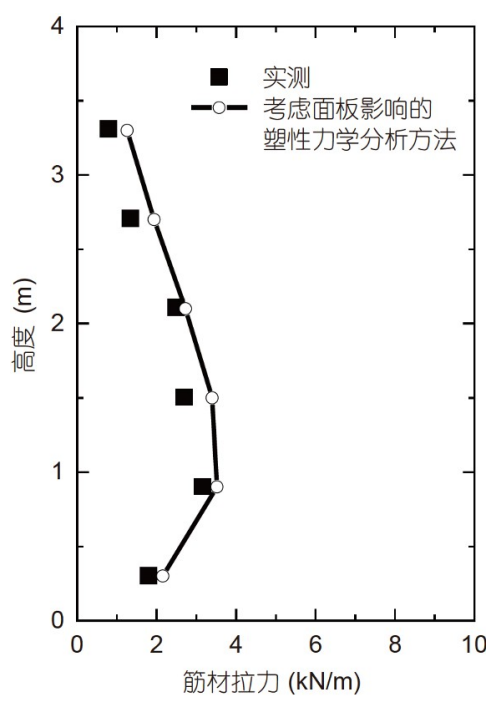

图 11 筋材拉力塑性分析结果与实测结果比较

Figure 11 Comparison of the measured and predicted results of the maximum reinforcement loads.

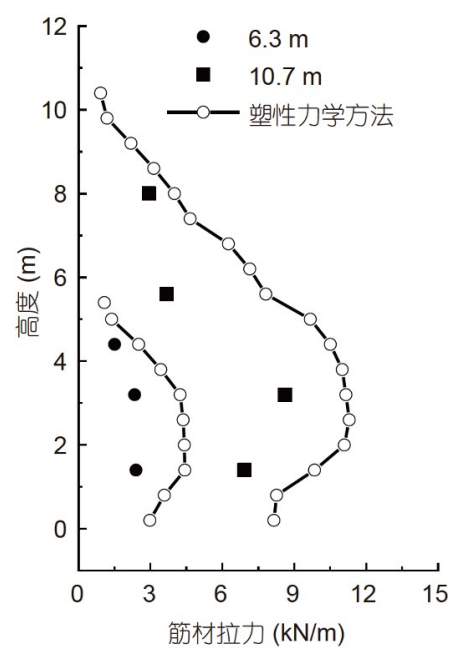

图 12 两组加筋土挡墙实测筋材拉力与塑性力学方法预测 拉力比较

Figure 12 Comparison of the measured and predicted results of the maximum reinforcement loads for two GRS retaining walls.

浇面板及连续钢筋混凝土预制面板，因它们的刚度比 较大, 对填土的约束作用较强, 本文的方法可能趋于 保守. 此外, 本文的方法仅限于分析潜在滑移面处的 筋材拉力, 对于一些较高的挡墙, 在一些部位面板-筋 材连接力可能较大，甚至大于潜在滑移面处的筋材拉 力. 如何在简化分析方法中考虑这些因素, 需要进一 步深入研究予以解决. 
1 Li G X. From “Xi-rang” to geosynthetics (in Chinese). Chin J Geotech Eng, 2013, 35: 144-149 [李广信. 从息壤到土工合成材料. 岩土工程学 报, 2013, 35: 144-149]

2 Li G X. The textual research about Xirang (in Chinese). China Civil Eng J, 2011, S2: 1-4 [李广信. 息壤考. 土木工程学报, 2011, S2: 1-4]

3 Zhang F, Zhu Y M, Yang S C, et al. Emission mitigation analysis of geosynthetic-reinforced walls (in Chinese). J Jilin Univ (Eng Tech Ed), 2021, 51: 631-637 [张飞, 朱玉明, 杨尚川, 等. 加筋土挡墙碳排放计算方法与减排性分析. 吉林大学学报(工学版), 2021, 51: 631-637]

4 Touze N. Healing the world: A geosynthetics solution. Geosynths Int, 2020, 28: 1-31

5 Berg R R, Christopher B R, Samtani N C. Design and Construction of Mechanically Stabilized Earth Walls and Reinforced Soil Slopes. Federal Highway Administration. Publication No. FHWA-NHI-10-024. 2009

6 British Standard BS8006-1. Code of Practice for Strengthened/Reinforeced Soils and Other Fills. London: British Standards Institution, 2010

7 NCMA. Design Manual for Segmental Retaining Walls. 3rd ed. Washington DC: National Concrete Masonry Association, 2010

8 Yang G Q, Xu C, Zhang M X, et al. Geosynthetics Reinforcement Soil Structure Application Guidance (in Chinese). Beijing: China Communications Press, 2016 [杨广庆, 徐超, 张孟喜, 等. 土工合成材料加筋土结构应用技术指南. 北京: 人民交通出版社. 2016]

9 Jiang Y, Han J, Zornberg J, et al. Numerical analysis of field geosynthetic-reinforced retaining walls with secondary reinforcement. Géotechnique, 2019, 69: 122-132

10 Yu Y, Bathurst R J, Allen T M. Numerical modelling of two full-scale reinforced soil wrapped-face walls. Geotext Geomembranes, 2017, 45: 237-249

11 Chen J F, Tian D, Liu J X. Internal failure mechanism of reinforced soil walls with flexible/rigid facings (in Chinese). Rock Soil Mech, 2018, 39: 2353-2360 [陈建峰, 田丹, 柳军修. 刚/柔性组合墙面加筋土挡墙内部破坏机制. 岩土力学, 2018, 39: 2353-2360]

12 Zhang Z C, Chen Y M. Model test and numerical analysis of micropile-MSE wall (in Chinese). Chin J Rock Mech Eng, 2017, 36: 987-996 [张智 超, 陈育民. 微型桩-加筋土挡墙的模型试验和数值模拟分析. 岩石力学与工程学报, 2017, 36: 987-996]

13 Chen J F, Zhang W. Numerical modeling of a reinforced soil segmental retaining wall designed using the K-stiffness method (in Chinese). Chin J Geotech Eng, 2017, 39: 1004-1011 [陈建峰, 张琬. 采用K-刚度法设计的模块式加筋土挡墙数值模拟. 岩土工程学报, 2017, 39: 1004-1011]

14 Zhang Y, Wang L, Liu H B. Influence of facing batter angle on reinforcement load of reinforced soil retaining wall with modular block facing (in Chinese). Chin J Geotech Eng, 2017, 39: 1680-1688 [张垭, 汪否, 刘华北. 面板倾角对模块式面板加筋土挡墙筋材内力的影响. 岩土工程学 报, 2017, 39: 1680-1688]

15 Zhu H W, Yao L K, Chen X L, et al. Seismic behaviors and design recommendations of ecological bag-reinforced retaining wall (in Chinese). Chin J Geotech Eng, 2017, 39: 319-326 [朱宏伟, 姚令㑆, 陈晓龙, 等. 土工格栅返包土工袋加筋土挡墙的地震响应分析及设计建议. 岩土工 程学报, 2017, 39: 319-326]

16 Helwany M B, Wu J T H. A numerical model for analyzing long-term performance of geosynthetic-reinforced soil structures. Geosynths Int, 1995, 2: 429-453

17 Liu H, Wang X, Song E. Long-term behavior of GRS retaining walls with marginal backfill soils. Geotext Geomembranes, 2009, 27: 295-307

18 Liu H, Won M S. Long-term reinforcement load of geosynthetic-reinforced soil retaining walls. J Geotech Geoenviron Eng, 2009, 135: 875-889

19 Liu H, Martinez J. Creep behaviour of sand-geomembrane interfaces. Geosynths Int, 2014, 21: 83-88

20 Allen T M, Bathurst R J. Observed long-term performance of geosynthetic walls and implications for design. Geosynths Int, 2002, 9: 567-606

21 Allen T M, Bathurst R J. Design and performance of 6.3-m-high, block-faced geogrid wall designed using K-stiffness method. J Geotech Geoenviron Eng, 2014, 140: 04013016

22 Allen T M, Bathurst R J. Performance of an $11 \mathrm{~m}$ high block-faced geogrid wall designed using the K-stiffness method. Can Geotech J, 2014, 51: $16-29$

23 Bathurst R J, Miyata Y, Nernheim A, et al. Refinement of K-stiffness method for geosynthetic-reinforced soil walls. Geosynths Int, 2008, 15: 269-295

24 Leshchinsky D. On global equilibrium in design of geosynthetic reinforced walls. J Geotech Geoenviron Eng, 2009, 135: 309-315

25 Leshchinsky D, Vahedifard F. Impact of toe resistance in reinforced masonry block walls: Design dilemma. J Geotech Geoenviron Eng, 2012, 138: $236-240$ 
26 Mirmoradi S H, Ehrlich M. Numerical evaluation of the behavior of GRS walls with segmental block facing under working stress conditions. J Geotech Geoenviron Eng, 2015, 141: 04014109

27 Mirmoradi S H, Ehrlich M. Effects of facing, reinforcement stiffness, toe resistance, and height on reinforced walls. Geotext Geomembranes, 2017, 45: 67-76

28 Ehrlich M, Mitchell J K. Working stress design method for reinforced soil walls. J Geotech Engrg, 1994, 120: 625-645

29 Ehrlich M, Mirmoradi S H. Evaluation of the effects of facing stiffness and toe resistance on the behavior of GRS walls. Geotext Geomembranes, 2013, 40: $28-36$

30 Liu H, Won M S. Stress dilatancy and reinforcement load of vertical-reinforced soil composite: Analytical method. J Eng Mech, 2014, 140: 630639

31 Liu H. Reinforcement load and compression of reinforced soil mass under surcharge loading. J Geotech Geoenviron Eng, 2015, 141: 04015017

32 Liu H, Yang G, Hung C. Analyzing reinforcement loads of vertical geosynthetic-reinforced soil walls considering toe restraint. Int J Geomech, 2017, 17: 04016140

33 Liu H. Required reinforcement stiffness for vertical geosynthetic-reinforced-soil walls at strength limit state. Géotechnique, 2016, 66: 424-434

34 Wang L, Liu H, Wang C. Earth pressure coefficients for reinforcement loads of vertical geosynthetic-reinforced soil retaining walls under working stress conditions. Geotext Geomembranes, 2018, 46: 486-496

35 Liu H B, Wang L, Wang C H, et al. Analysis methods for the reinforcement loads of geosynthetic-reinforced soil retaining walls (in Chinese). Eng Mech, 2017, 34: 1-11 [刘华北, 汪否, 王春海, 等. 土工合成材料加筋土挡墙筋材内力分析. 工程力学, 2017, 34: 1-11]

36 Shibuya S, Tatsuoka F, Teachavorasinskun S, et al. Elastic deformation properties of geomaterials. Soils Found, 1992, 32: 26-46

37 Gu X Q, Yang J, Huang M S, et al. Combining bender element, resonant column and cyclic torsional shear tests to determine small strain shear modulus of sand (in Chinese). Chin J Geotech Eng, 2016, 38: 740-746 [顾晓强, 杨峻, 黄茂松, 等. 砂土剪切模量测定的弯曲元、共振柱和循 环扭剪试验. 岩土工程学报, 2016, 38: 740-746]

38 Peng F L, Li F L, Li J Z, et al. Loading rate effects on stress-strain relation of sandy soils (in Chinese). Rock Soil Mech, 2007, 28(s1): 16-20 [彭 芳乐, 李福林, 李建中, 等. 砂土应力-应变关系的加载速率效应分析. 岩土力学, 2007, 28(s1): 16-20]

39 Zhang Y, Zhang B Y, Li G X, et al. Combined tension-compression triaxial tests and extended Duncan-Chang model of compacted clay (in Chinese). Chin J Geotech Eng, 2010, 32: 999-1004 [张琰, 张丙印, 李广信, 等. 压实黏土拉压组合三轴试验和扩展邓肯张模型. 岩土工程学 报, 2010, 32: 999-1004]

40 Krabbenhoft K, Wang J. Analytical stress-strain relation for soils. Can Geotech J, 2021, doi: 10.1139/cgj-2021-0043

41 Hatami K, Bathurst R J. Development and verification of a numerical model for the analysis of geosynthetic-reinforced soil segmental walls under working stress conditions. Can Geotech J, 2005, 42: 1066-1085

42 Hatami K, Bathurst R J. Numerical model for reinforced soil segmental walls under surcharge loading. J Geotech Geoenviron Eng, 2006, 132: 673-684

43 Tatsuoka F, Siddiquee M S A, Park C S, et al. Modelling stress-strain relations of sand. Soils Found, 1993, 33: 60-81

44 Kongkitkul W, Tatsuoka F, Hirakawa D. Effects of reinforcement type and loading history on the deformation of reinforced sand in plane strain compression. Soils Found, 2007, 47: 395-414

45 Duncan J M, Byrne P, Wong K S, et al. Strength, stress-strain and bulk modulus parameters for finite element analysis of stress and movements in soil masses. Berkeley, CA: University of California, College of Engineering, 1980. Report No. UCB/GT/80-01

46 Laman M, Yildiz A. Numerical studies of ring foundations on geogrid-reinforced sand. Geosynths Int, 2007, 14: 52-64

47 Wang L, Wang C, Liu H, et al. One-step analytical method for required reinforcement stiffness of vertical reinforced soil wall with given factor of safety on backfill soil. Geotext Geomembranes, 2021, 49: 343-350

48 Nascimento G, Ehrlich M, Mirmoradi S H. Numerical-simulation of compaction-induced stress for the analysis of RS walls under surcharge loading. Geotext Geomembranes, 2020, 48: 532-538

49 Huang B, Bathurst R J, Hatami K, et al. Influence of toe restraint on reinforced soil segmental walls. Can Geotech J, 2010, 47: 885-904

50 Chen J, Yu Y, Bathurst R J. Influence of leveling pad interface properties on soil reinforcement loads for walls on rigid foundations. In: GeoShanghai 2014. Shanghai: Geotechnical Special Publication. 481-492

51 Nelson R D. Performance of two full-scale geosynthetic reinforced retaining walls-modular block and incremental panel. Dissertation for Master's Degree. Ontario: Royal Military College, 2005 
52 Yu Y, Bathurst R J, Allen T M. Numerical modeling of the SR-18 geogrid reinforced modular block retaining walls. J Geotech Geoenviron Eng, 2016, 142: 04016003

\title{
Analyzing operational reinforcement loads of geosynthetic-reinforced soil retaining walls based on plasticity theory
}

\author{
LIU HuaBei ${ }^{1}$, WANG ChunHai ${ }^{1} \&$ WANG Lei ${ }^{2}$ \\ ${ }^{1}$ School of Civil and Hydraulic Engineering, Huazhong University of Science and Technology, Wuhan 430074, China; \\ ${ }^{2}$ School of Software Engineering, Jiangxi University of Science and Technology, Nanchang 330031, China
}

Geosynthetic-reinforced soil (GRS) retaining walls have exhibited good performance in extensive engineering applications. Compared with conventional earth-retaining structures, GRS retaining walls can considerably reduce the use of Portland cement and steel reinforcements, which in turn leads to a reduction in $\mathrm{CO}_{2}$ emissions. Currently, the design of GRS retaining walls primarily focuses on the strength design and consists of various stability checks. However, this type of soil structure is flexible by nature, and its serviceability design may be as important as its strength design. Because few methods are available for serviceability analyses, over the last decade, in-depth investigations into simplistic approaches have been carried out to analyze reinforcement loads under working stress conditions. The proposed approach is based on soil-reinforcement compatible deformations at the internal potential failure surface and incorporates the nonlinear stress-strain relationships between the backfill materials, their plastic deformation, the longterm stiffness of the geosynthetic reinforcement, the force equilibrium of the active soil mass, and the force equilibrium of the facing units. The proposed approach was validated against a series of measured reinforcement loads and the results from calibrated numerical analyses. It is shown that the proposed approach has the capacity to satisfactorily estimate the reinforcement loads of vertical and batter reinforced soil retaining walls with concrete block facings.

geosynthetics, reinforced soil retaining walls, serviceability, reinforcement loads, theory of plasticity

doi: $10.1360 /$ SST-2021-0287 